\title{
Fine-Scale Adaptations to Environmental Variation and Growth Strategies Drive Phyllosphere Methylobacterium Diversity
}

\author{
aDépartement de Sciences Biologiques, Université de Montréal, Montreal, Quebec, Canada \\ bDépartement des Sciences Biologiques, Université du Québec à Montréal, Montreal, Quebec, Canada \\ cDepartment of Biological Sciences, University of Idaho, Moscow, Idaho, USA \\ dDepartment of Biology, McGill University, Montreal, Quebec, Canada
}

(D) Jean-Baptiste Leducq, ${ }^{\mathrm{a} b \mathrm{~b}, \mathrm{c}}$ Émilie Seyer-Lamontagne, ${ }^{a}$ Domitille Condrain-Morel, ${ }^{\mathrm{b}}$ Geneviève Bourret, ${ }^{\mathrm{b}}$ David Sneddon, ${ }^{\mathrm{c}}$

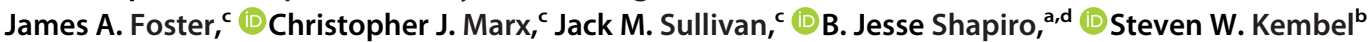

ABSTRACT Methylobacterium is a prevalent bacterial genus of the phyllosphere. Despite its ubiquity, little is known about the extent to which its diversity reflects neutral processes like migration and drift, versus environmental filtering of life history strategies and adaptations. In two temperate forests, we investigated how phylogenetic diversity within Methylobacterium is structured by biogeography, seasonality, and growth strategies. Using deep, culture-independent barcoded marker gene sequencing coupled with culture-based approaches, we uncovered a considerable diversity of Methylobacterium in the phyllosphere. We cultured different subsets of Methylobacterium lineages depending upon the temperature of isolation and growth $\left(20^{\circ} \mathrm{C}\right.$ or $\left.30^{\circ} \mathrm{C}\right)$, suggesting long-term adaptation to temperature. To a lesser extent than temperature adaptation, Methylobacterium diversity was also structured across large ( $>100 \mathrm{~km}$; between forests) and small $(<1.2 \mathrm{~km}$; within forests) geographical scales, among host tree species, and was dynamic over seasons. By measuring the growth of 79 isolates during different temperature treatments, we observed contrasting growth performances, with strong lineage- and seasondependent variations in growth strategies. Finally, we documented a progressive replacement of lineages with a high-yield growth strategy typical of cooperative, structured communities in favor of those characterized by rapid growth, resulting in convergence and homogenization of community structure at the end of the growing season. Together, our results show how Methylobacterium is phylogenetically structured into lineages with distinct growth strategies, which helps explain their differential abundance across regions, host tree species, and time. This work paves the way for further investigation of adaptive strategies and traits within a ubiquitous phyllosphere genus.

IMPORTANCE Methylobacterium is a bacterial group tied to plants. Despite the ubiquity of methylobacteria and the importance to their hosts, little is known about the processes driving Methylobacterium community dynamics. By combining traditional culture-dependent and -independent (metabarcoding) approaches, we monitored Methylobacterium diversity in two temperate forests over a growing season. On the surface of tree leaves, we discovered remarkably diverse and dynamic Methylobacterium communities over short temporal (from June to October) and spatial (within $1.2 \mathrm{~km}$ ) scales. Because we cultured different subsets of Methylobacterium diversity depending on the temperature of incubation, we suspected that these dynamics partly reflected climatic adaptation. By culturing strains under laboratory conditions mimicking seasonal variations, we found that diversity and environmental variations were indeed good predictors of Methylobacterium growth performances. Our findings suggest that Methylobacterium community dynamics at the surface of tree leaves results from the succession of strains with contrasting growth strategies in response to environmental variations.
Editor Paul Keim, Northern Arizona University Copyright $\odot 2022$ Leducq et al. This is an open-access article distributed under the terms of the Creative Commons Attribution 4.0 International license.

Address correspondence to Jean-Baptiste Leduca jeanbaptiste@uidaho.edu, B. Jesse Shapiro, jesse.shapiro@mcgill.ca, or Steven W. Kembel, kembel.steven_w@uqam.ca. The authors declare no conflict of interest. Received 25 October 2021 Accepted 23 November 2021 Published 25 January 2022 
KEYWORDS Methylobacterium diversity, phyllosphere community dynamics, rpoB barcoding, temperature adaptation, growth strategies in Bacteria, Methylobacterium, microbial communities, phyllosphere-inhabiting microbes

T he phyllosphere, the aerial parts of plants including leaves, is a microbial habitat estimated to be as vast as twice the surface of the earth (1). Although exposed to harsh conditions, including UV radiation, temperature variation, and poor nutrient availability, the phyllosphere harbors a diverse community of microorganisms, of which bacteria are the most abundant (1). A key challenge in microbial ecology and evolution is understanding the evolutionary and ecological processes that maintain diversity in habitats such as the phyllosphere. Bacteria living in the phyllosphere carry out key functions, including nitrogen fixation, growth stimulation, and protection against pathogens (1-3). At broad spatial and temporal scales, bacterial diversity in the phyllosphere varies as a function of geography and host plant species, potentially due to restricted migration and local adaptation to the biotic and abiotic environment (4-6), leading to patterns of cophylogenetic evolutionary association between phyllosphere bacteria and their host plants (7). Whether those eco-evolutionary processes are important at the scale of several days to several years, as microbes and their host plants migrate and adapt to changing climates, is still an open question (8). Another challenge is to link seasonal variation with plant-associated microbial community dynamics, as shifts in microbial community composition are tightly linked with host plant carbon cycling (9) and ecosystem functions, including nitrogen fixation (10). More generally, we understand very little about how the ecological strategies of phyllosphere bacteria vary among lineages and in response to variation in environmental conditions throughout the growing season $(9,11)$.

Phenotypic traits are often phylogenetically conserved in microbes (12), and these traits influence the assembly of ecological communities through their mediation of organismal interactions with the abiotic and biotic environment (13). Recent work has shown that many microbial traits exhibit a phylogenetic signal, with closely related lineages possessing more similar traits, although the phylogenetic depth at which this signal is evident differs among traits (14). Most comparative studies of microbial trait evolution have focused on broad patterns across major phyla and classes (14), although some studies have found evidence for complex patterns of biotic and abiotic niche preferences evolving within genus-level phylogenies $(15,16)$. Furthermore, to date, the majority of studies of the diversity of plant-associated microbes have been based on the use of universal marker genes such as the bacterial $16 \mathrm{~S}$ rRNA gene, providing a global picture of long-term bacterial adaptation to different biomes and host plants at broad phylogenetic scales (17). However, these studies lack sufficient resolution to assess the evolutionary processes at finer spatial and temporal scales that lead to the origin of adaptations within microbial genera and species $(18,19)$.

The Rhizobiales genus Methylobacterium (Alphaproteobacteria, Rhizobiales, Methylobacteriaceae) is one of the most prevalent bacterial genera of the phyllosphere, present on nearly every plant (20-22). Characterized by pink colonies due to carotenoid production, methylobacteria are facultative methylotrophs, able to use one-carbon compounds, such as methanol excreted by plants, as sole carbon sources $(23,24)$. Experimental studies have shown the important roles of Methylobacterium in plant physiology, including growth stimulation through hormone secretion (25-27), heavy metal sequestration (27), antiphytopathogenic compound secretion, and nitrogen fixation in plant nodules (28), sparking increasing interest in the use of Methylobacterium in plant biotechnology applications $(27,29,30)$. Although up to 64 Methylobacterium species have been described (31-39), genomic and phenotypic information was until recently limited to a small number of model species: M. extorquens, M. populi, M. nodulans, M. aquaticum, and $M$. radiotolerans, mostly isolated from anthropogenic environments and only rarely from plants (40-44). Additionally, Methylobacterium was mostly isolated assuming that its optimal growth was in the range of 25 to $30^{\circ} \mathrm{C}$ (45), an approach 
that could bias strain collections toward mesophyllic isolates to the exclusion of isolates from temperate forests, where temperatures typically range from 10 to $20^{\circ} \mathrm{C}$ during the growing season (46). Newly available genomic and metagenomic data now allow a better understanding of the distribution of Methylobacterium diversity across biomes (31) and suggest that they represent a stable and diverse fraction of the phyllosphere microbiota (22). However, we still understand relatively little about the drivers of the evolution and adaptation of Methylobacterium in natural habitats.

In this study, we assessed the diversity of Methylobacterium in temperate forests and asked whether methylobacteria associated with tree leaves act as a single unstructured population, or if their diversity is structured by regional factors (e.g., a combination of isolation by distance and regional environmental variation) or by niche adaptation (e.g., host tree or temperature adaptation) (12). First, we assessed Methylobacterium diversity by combining culturing and metabarcoding approaches along with phylogenetic analysis and quantified how this diversity varied across space, time, and environment in the phyllosphere. Second, we quantified the extent of phylogenetic niche differentiation within the genus, with a focus on quantifying the evidence for adaptation to local environmental variation at different spatial, temporal, and phylogenetic scales. We hypothesized that distinct phylogenetic lineages would be associated with distinct environmental niches. Third, we quantified Methylobacterium growth performance under fine-scale environmental variation, with a focus on temperature, to determine whether fine-scale changes in diversity over space and time might result from environmental filtering of isolates with contrasting growth strategies under local environmental conditions. We found that Methylobacterium phyllosphere diversity consisted of deeply branching phylogenetic lineages associated with distinct growth phenotypes, isolation temperatures, and large-scale spatial effects (forest of origin), while finer-scale spatial effects, host tree species, and time of sampling were more weakly and shallowly phylogenetically structured. Over the course of a year, from spring to fall, we observed a homogenization of Methylobacterium community structure coinciding with the progressive replacement of isolates with a high-yield strategy by isolates with rapid growth. Together, our results show that this ubiquitous phyllosphere genus is structured into lineages with distinct growth strategies, which helps explain their differential abundance across space and time.

\section{RESULTS}

Phylogenetics of plant-associated Methylobacterium diversity. A phylogeny of 153 Methylobacterium isolates built from available genomic databases showed that plants (65\% of isolates) and especially the phyllosphere compartment ( $41 \%$ of isolates) were the most prevalent source of Methylobacterium sampled to date (Fig. 1; see Data Set S1a in the supplemental material). Phyllosphere-associated diversity was not randomly distributed in the Methylobacterium phylogenetic tree. Isolates from the phyllosphere represented the largest part of diversity within group A ( $56 \%$ of isolates) but not in groups B and C (17\% and $12 \%$ of isolates, respectively). Group A was paraphyletic, and most of its diversity consisted of undescribed taxa falling outside previously well-described lineages. Accordingly, we subdivided Methylobacterium group A into 9 monophyletic clades (A1 to A9).

16S rRNA community analyses of the tree phyllosphere. We focused on Methylobacterium phyllosphere diversity variation observable at the scale of seasonal variation (within the year 2018) on individual trees within two temperate forests of northeastern North America (Fig. 2a and b; Data Set S1b and g): Mont Saint Hilaire (MSH) (Fig. 2c) and Station Biologique des Laurentides (SBL) (Fig. 2d). The distribution of the phyllosphere bacterial community assessed in 46 leaf samples by bacterial $16 \mathrm{~S}$ rRNA gene amplicon sequence variants (ASVs) was mostly explained by differences among forests (31.6\% of variation explained; $P<0.001$; peremutational multivariate analysis of variance [PERMANOVA]), host tree species (15.6\% of variation; $P<0.001$ ), and time of sampling (12.0\%; $P<0.05)$ (Table 1$)$. Although representing only $1.3 \%(0.0$ to $3.2 \%$ per sample) of total $16 \mathrm{~S}$ rRNA gene sequence diversity, Methylobacterium was 


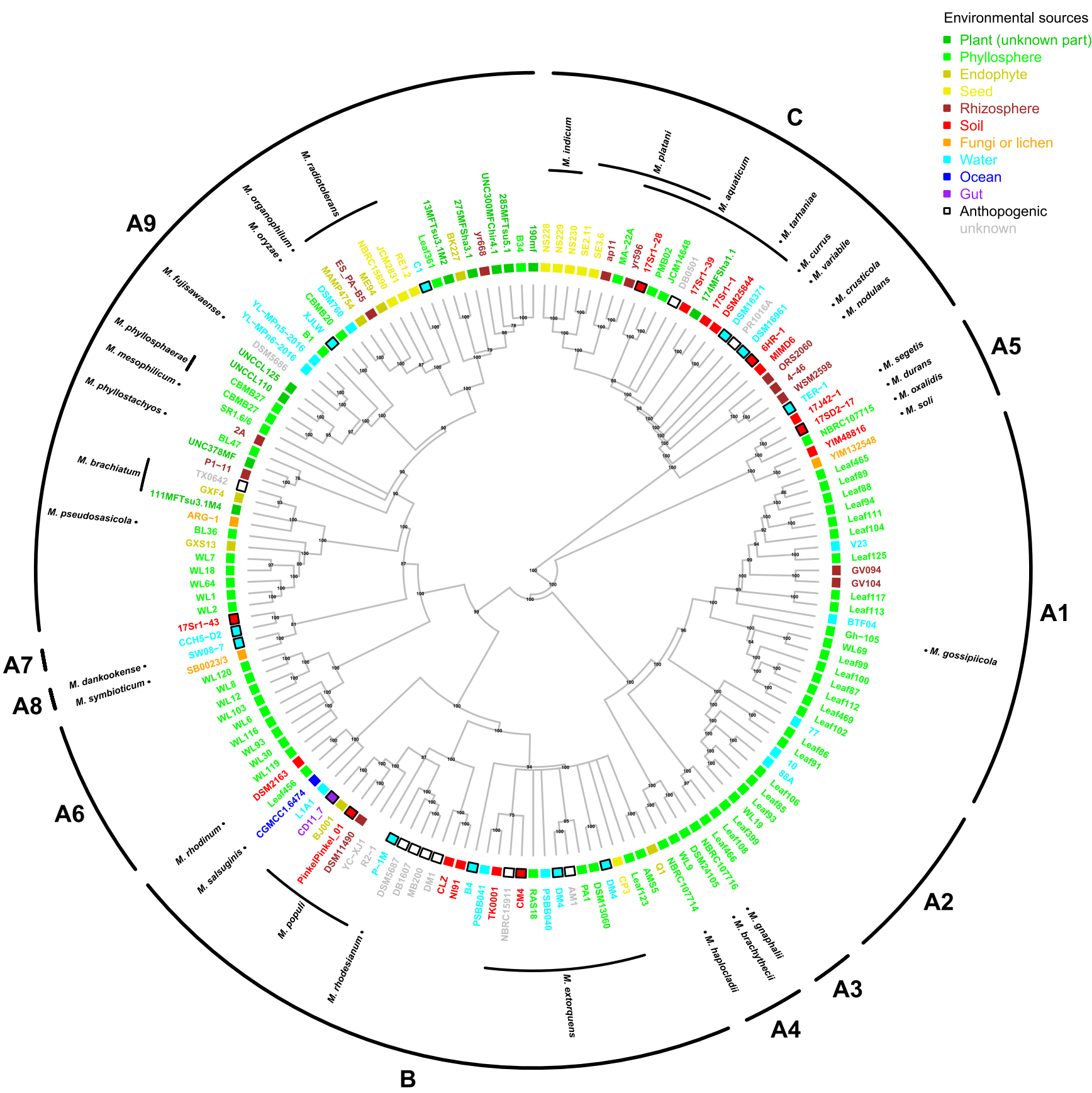

FIG 1 Methylobacterium phylogeny and ecology. Most of Methylobacterium diversity is found in association with plants, especially in the phyllosphere. Phylogenetic consensus tree (nodal posterior probabilities indicated next to the branches) from rpoB complete nucleotide sequences available for 153 Methylobacterium genomes and rooted on 32 Methylobacteriaceae outgroups (Microvirga and Enterovirga not shown) (see Data Set S1a). For each genome, the species name, anthropogenic origin (black squares), and/or environmental origin (color code on top right) are indicated. Groups A, B, and C are adapted from a report by Green and Ardley (31).

present in almost all analyzed samples (45 out of 46) (Data Set S1h). We assigned the 15 Methylobacterium ASVs identified by $16 \mathrm{~S}$ rRNA gene sequencing to clades from Methylobacterium group A: A9 (related to M. phyllosphaerae/M. mesophilicum/M. phyllostachyos/M. pseudosasicola/M. organophilum; $0.87 \%$ of total diversity, nine ASVs), A6 (related to M. cerastii, $0.29 \%$; one ASV), and A1 (related to M. gossipicola; $0.13 \%$, three ASVs) (Table S2; Data Set S1i). With two rare ASVs $(<0.01 \%$ of relative abundance) related to $M$. komagatae, belonging to group A (31) but unrelated to any aforementioned clade, we defined a new clade (A10). No ASVs from MSH or SBL were assigned to group B or group C. 
a

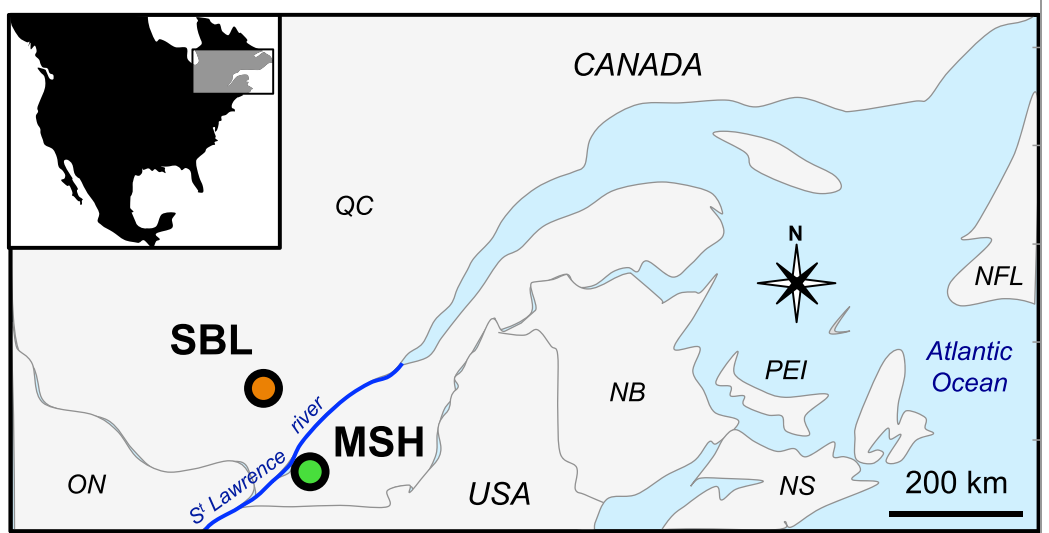

C

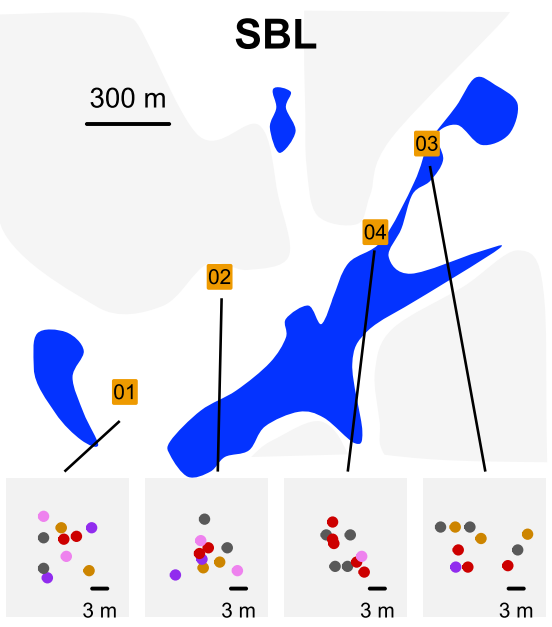

b

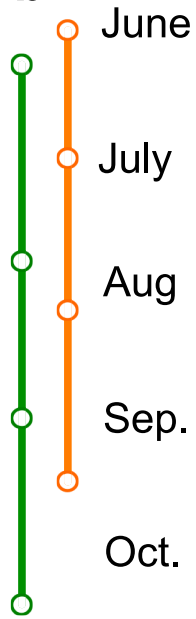

FIG 2 Sampling design. (a) Locations of the two sampled forests, MSH (green) and SBL (orange), in the province of Québec (Canada). (b) Time line survey in each forest in 2018 ( 2 to 4 time points available per tree). (c and d) Detailed map of each forest and each plot within the forests (squares; 6 to 10 trees were sampled per plot) (see Data Set S1b in the supplemental material). For each plot, trees are indicated by points colored according to their taxonomy (color code on bottom right): ABBA, Abies balsamea; ACRU, Acer rubrum; ACSA, Acer saccharum; OSVI, Ostrya virginiana; QURU, Quercus rubra; FAGR, Fagus grandifolia; ASPE, Acer pensylvanicum. Shades of gray indicate elevation (50-m elevation scale).

Culture-based assessment of Methylobacterium diversity in the tree phyllosphere.

We evaluated the culturable part of Methylobacterium diversity from a subsample of 36 trees (18 per forest). Using $r p o B$ gene partial nucleotide sequences as a marker, we identified 167 pink isolates that we assigned to Methylobacterium based upon their phylogenetic placement (Data Set S1e and f; Fig. 3). As observed for 16S rRNA gene ASVs, most isolates were assigned to clades from group A typical of the phyllosphere: A9 (59.9\% of isolates), A6 (24.6\%), A1 (5.4\%), A10 (3.6\%), and A2 (related to M. bullatum and $M$. marchantiae) (1.8\%) (Data Set S1d). Few isolates were assigned to group $B$ ( $4.2 \%$ of isolates, related to $M$. extorquens) and none to group $C$ (Table S2). The higher polymorphism in the $r p o B$ marker revealed a considerable diversity within clades, as we identified 71 unique $r p o B$ sequences, in contrast to the smaller number obtained with $16 \mathrm{~S}$ rRNA gene barcoding (15 ASVs). We determined that Methylobacterium diversity assessed at various depths in the $r p o B$ phylogeny was systematically explained by the forest of origin $(4.5 \% \pm 1.0 \%$ of variance explained; PERMANOVA; $P<0.001)$ (Fig. 3a; Data Set S1j) and temperature of isolation $(5.9 \% \pm 2.1 \%$ of variance explained; $P<0.001)$. The temperature of isolation was the most important factor distinguishing 
TABLE 1 PERMANOVA analysis of variance in Bacteria and Methylobacterium community diversity $^{a}$

\begin{tabular}{|c|c|c|c|c|}
\hline \multirow[b]{2}{*}{ Factor or interaction } & \multicolumn{2}{|c|}{$\begin{array}{l}\text { Bacteria (16S rRNA gene) } \\
(n=46)\end{array}$} & \multicolumn{2}{|c|}{$\begin{array}{l}\text { Methylobacterium (rpoB) } \\
(n=179)\end{array}$} \\
\hline & $R^{2}$ & $P$ & $R^{2}$ & $P$ \\
\hline $\bar{F}$ & $0.316^{* * *}$ & $<0.000$ & $0.324^{* * *}$ & $<0.001$ \\
\hline $\mathrm{H}$ & $0.156^{* * *}$ & $<0.001$ & $0.071^{* * *}$ & $<0.001$ \\
\hline $\mathrm{D}$ & $0.120^{*}$ & 0.016 & $0.048^{* * *}$ & $<0.001$ \\
\hline$P$ & & & $0.080^{* * *}$ & $<0.001$ \\
\hline $\mathrm{F}: \mathrm{H}$ & 0.020 & 0.080 & 0.004 & 0.110 \\
\hline$H: D$ & 0.239 & 0.217 & $0.074^{* *}$ & 0.028 \\
\hline $\mathrm{H}: \mathrm{P}$ & & & $0.043^{* *}$ & 0.007 \\
\hline$D: P$ & & & 0.058 & 0.455 \\
\hline$H: D: P$ & & & 0.085 & 0.052 \\
\hline Residuals & 0.150 & & 0.213 & \\
\hline
\end{tabular}

apart of variance in dissimilarity $\left(R^{2}\right.$; Bray-Curtis index) and significance $(P ; P$ value) among samples is associated with four factors and their possible interactions $(F$, forest of origin; $D$, date of sampling; $H$, host tree species; $P$ plot within forest), and their significances are shown (10,000 permutations on ASV relative abundance, Hellinger transformation; ${ }^{* * *}, P<0.001 ;{ }^{* *}, P<0.01 ;{ }^{*}, P<0.05$ ). For the $16 \mathrm{~S}$ rRNA gene, plot within forests was omitted to conserve degrees of freedom. $n$, number of samples.

deep phylogenetic divergences (pairwise nucleotide similarity range, 0.948 to 0.993 ), while the forest of origin was slightly more important in structuring more recently diverged nodes (pairwise nucleotide similarity, $>0.993$ ). The time of sampling had a slight but significant effect on diversity $(2.1 \% \pm 0.2 \%$ of variance explained; $P<0.05)$, and it was observed only for higher pairwise nucleotide similarity values (range, 0.994 to 1.000). We did not observe any significant effects of host tree species on Methylobacterium isolate diversity, at any level of the phylogeny. In the phylogeny, we identified two nodes strongly associated with temperature of isolation, corresponding to clades $\mathrm{A} 6\left(20^{\circ} \mathrm{C} ; P<0.001\right.$; permutation test) and $\mathrm{A} 9$ and $\mathrm{A} 10\left(30^{\circ} \mathrm{C} ; P<0.001\right)$ (Fig. 3b). Other clades were evenly isolated at $20^{\circ} \mathrm{C}$ and $30^{\circ} \mathrm{C}$, and we observed no significant association between temperature of isolation and nodes embedded within clades. Nodes associated with the forest of origin also roughly corresponded to certain major clades, with clades $\mathrm{A} 1$ and $\mathrm{A} 2$ almost exclusively sampled in MSH $(P<0.01)$. Overall, clade A9 was isolated significantly more often at SBL $(P<0.001)$, but at least three of its subclades were significantly associated with either MSH or SBL $(P<0.05)$.

Comparison of Methylobacterium diversity assessed by rpoB barcoding and isolation. We performed culture-independent $r p o B$ amplicon sequencing for 179 leaf samples from 53 trees in both forests, allowing a monthly monitoring for most trees (Data Set S1b and g). We identified 283 Methylobacteriaceae rpoB ASVs in these samples (Data Set S1k and I), representing $24.6 \%$ of all sequences. Non-Methylobacteriaceae ASVs were mostly assigned to other Rhizobiales families ( 850 ASVs, $70.33 \%$ of sequence abundance) and to Caulobacterales (209 ASVs, $4.42 \%$ of sequence abundance) typical of the phyllosphere (Text S1), indicating that the rpoB marker can potentially be used at a broader taxonomic scale (Fig. S5a). Within Methylobacteriaceae, ASVs were mostly classified as Methylobacterium (200 ASVs, 23.05\% of sequence relative abundance) and Enterovirga (78 ASVs, 1.56\%) (Data Set S1k). We assigned most of Methylobacterium ASVs to previously cultured clades A9 (45.2\% of Methylobacterium sequence abundance), A6 (24.3\%), A1 (6.1\%), and A10 (1.0\%) (Data Set S1k, Table S2, and Fig. S5b). Estimates of Methylobacterium diversity based on $r p o B$ sequences from culture-independent sequencing were generally concordant with estimates based on 16S rRNA gene barcoding (Fig. S5c; Table S2) and estimates from cultured isolates (Fig. S5d; Table S2). The major exception was group B, representing $19.1 \%$ of Methylobacterium sequence abundance ( $r p o B$ barcoding) but not detected by $16 \mathrm{~S}$ rRNA gene barcoding and representing $4.2 \%$ of isolates (Table S2). Clade A4 (related to $M$. gnaphalii and $M$. brachytecii) represented $1.7 \%$ of Methylobacterium sequence abundance ( $r$ poB barcoding) but was not detected by $16 \mathrm{~S}$ rRNA gene barcoding, nor was it isolated. Other clades could be detected by rроB barcoding with low sequence 
a

b B

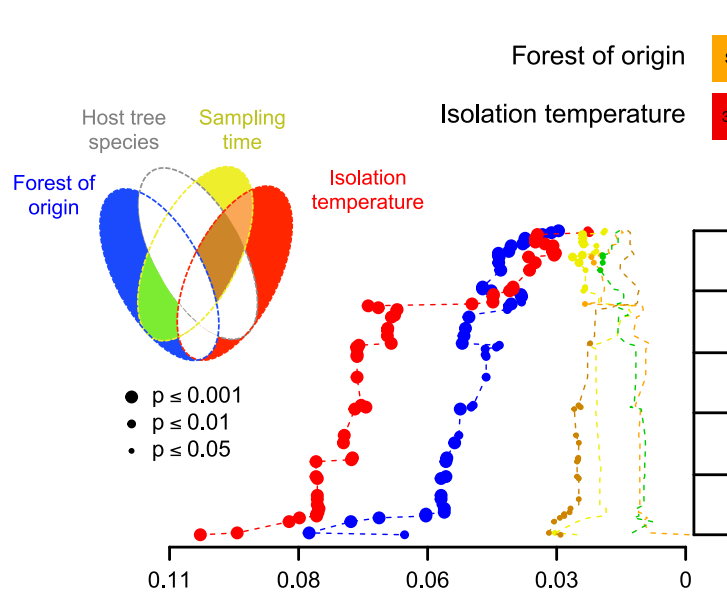

\begin{tabular}{l|l}
\hline $30^{\circ} \mathrm{C}$ & $20^{\circ} \mathrm{C}$ \\
\hline
\end{tabular}

\section{Proportion of variance}

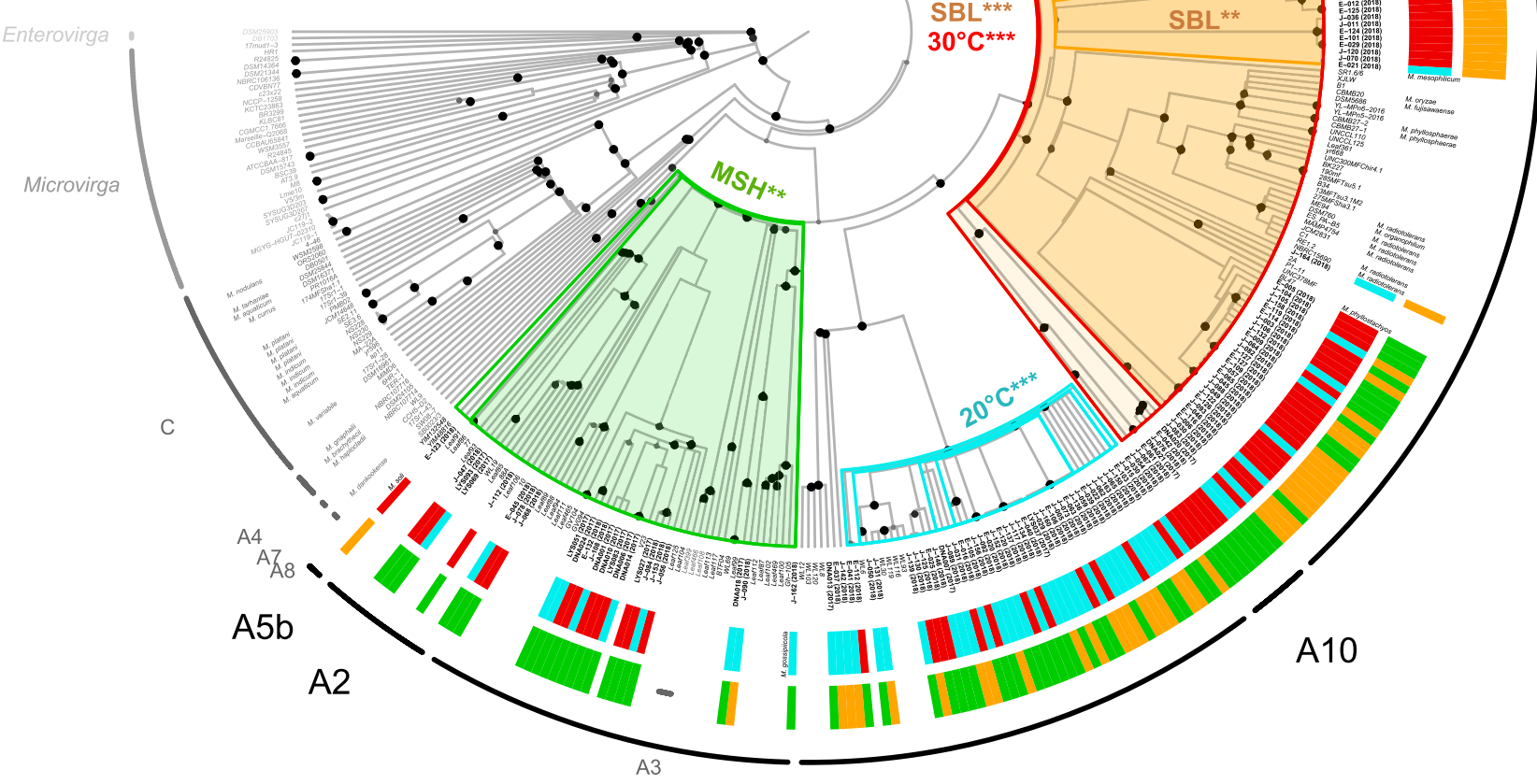

A1

A6

FIG 3 Tests for phylogenetic association of traits with culture-based estimation of Methylobacterium diversity. (a) Proportion of variance (PERMANOVA; $x$ axis) in Methylobacterium isolate diversity explained by forest of origin, host tree species, sampling date, temperature of isolation, and their interactions (see Venn diagram on top left for color code) in function of pairwise nucleotide similarity (PS; $y$ axis) (see Data set S1j) in a phylogenetic tree (partial rpoB nucleotide sequences of 187 isolates and 188 Methylobacteriaceae reference sequences). (b) Permutation test for node association with forest of origin and temperature of isolation (color code on top) mapped on the $r p o B$ phylogeny (scaled on PS values). Frames in the tree indicate nodes significantly associated with at least one factor (ANOVA; Bonferroni correction; $* * *$, $P<0.001 ;{ }^{* *}, P<0.01 ;{ }^{*}, P<0.05$ ). For each isolate (names in bold), colored boxes at the tip of the tree indicate forest of origin and temperature of isolation.

abundance $(<0.3 \%)$ but not by $16 \mathrm{~S}$ rRNA gene barcoding and were unevenly isolated $(<1.8 \%$ of isolates).

Fine-scale temporal and spatial distribution of Methylobacterium diversity assessed by rров barcoding. The community composition of the 200 Methylobacterium ASVs was mostly explained by spatial variation at both large (distance between forests, $100 \mathrm{~km}$ ) and local (distance between plots within forest, 150 to 1,200 m) scales, as well as sampling date during the growing season ( 1 to 5 months) (proportion of variation explained, 32.4\%, 8.0\%, and 4.8\%, respectively; $P<0.001$; PERMANOVA) (Table 1). We 


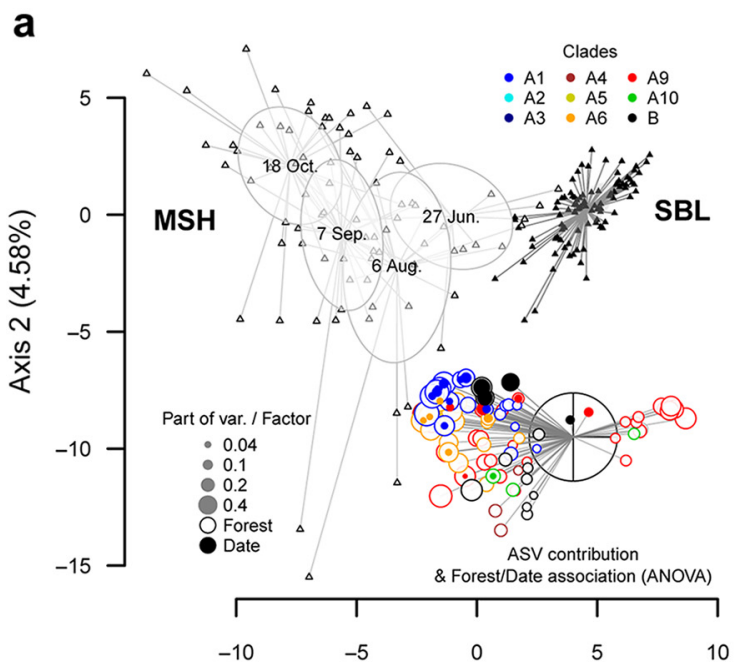

Axis 1 (15.38\%)

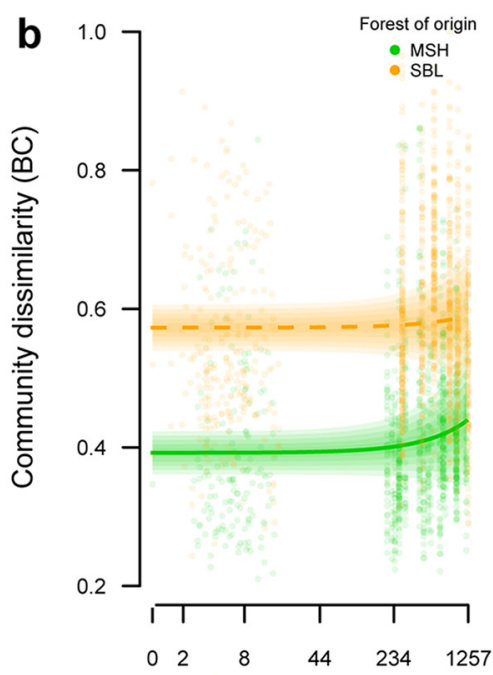

pDistance (log scale, $\mathrm{m}$ )

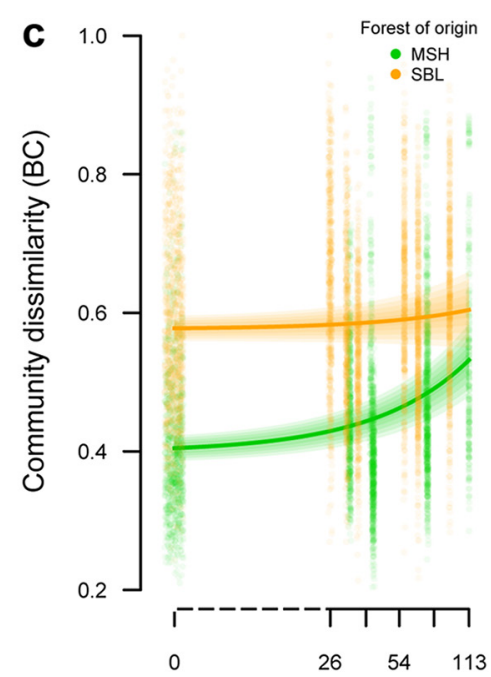

pTime (log scale, days)
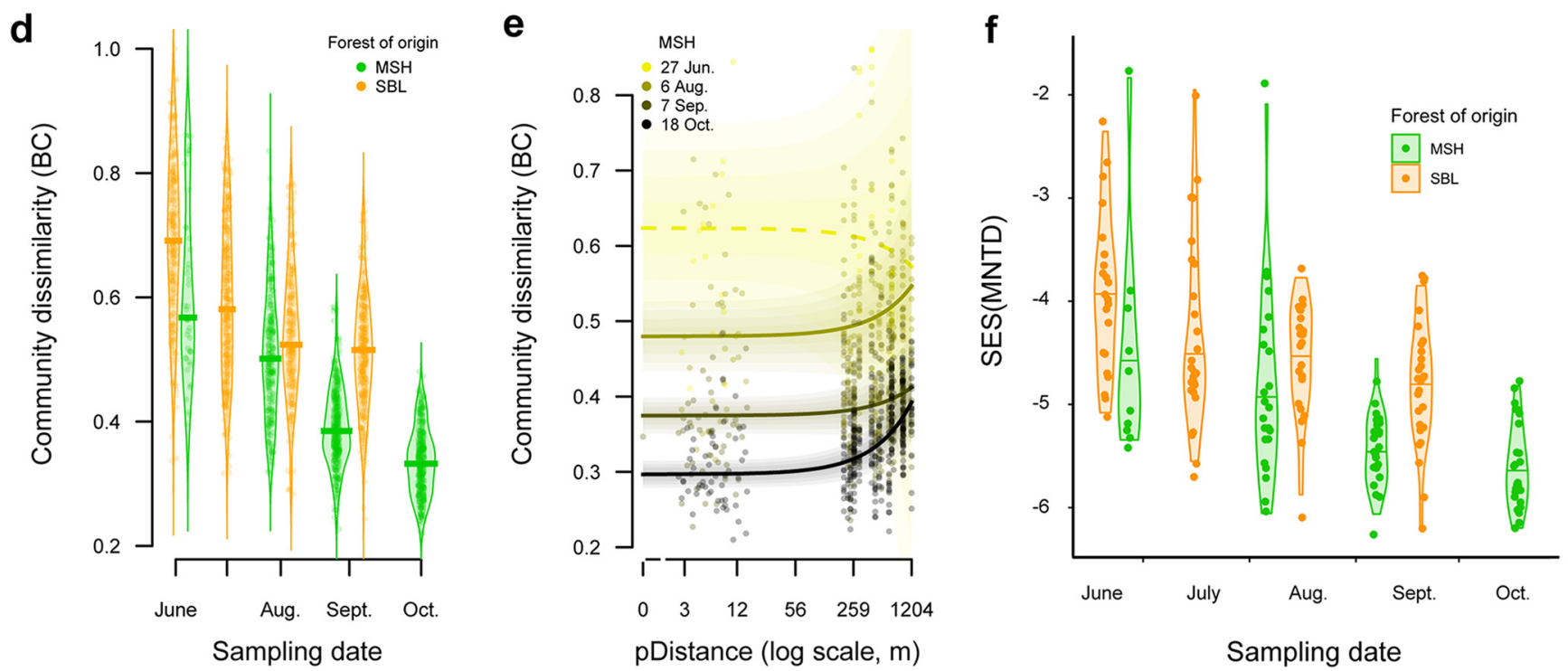

FIG 4 Short-scale spatial and temporal dynamics of Methylobacterium communities assessed by rpoB barcoding. (a) A principal-component analysis (PCA) of Methylobacterium ASV relative abundance shows that 179 phyllosphere samples cluster according to forest of origin (MSH, open triangles; SBL, filled triangles) and date of sampling (detail shown only for MSH). The significant association of 83 and 25 ASVs with forest of origin and/or sampling date, respectively, is shown (points colored according to clade assignation; legend on top right). (b and c) Spatial (b) and temporal (c) autocorrelation analyses conducted in each forest separately. Points represent Bray-Curtis (BC) dissimilarity in function of pairwise geographic (pDist; b) or pairwise time (pTime; $c$ ) distance separating two communities. For each forest and variable, the predicted linear regression is indicated (solid line, $P<0.001$; dotted line, $P>0.05$; ANOVA). (d) BC dissimilarity in function of sampling time for each forest. (e) Detail of spatial autocorrelation analyzes in MSH, conducted for each sampling time point separately. ( $f$ ) Standardized effect size of mean nearest taxon phylogenetic distance [SES(MNTD)] between forests and across sampling dates. Negative values of SES(MNTD) indicate communities contain ASVs that are phylogenetically clustered compared to a null model of stochastic community assembly.

observed slight but significant effects of host tree species and of the interaction between host tree species and plots within forests on Methylobacterium community composition (explaining $7.1 \%$ and $4.3 \%$ of variation in community composition; $P<0.001$ and $P<0.01$, respectively; PERMANOVA) (Table 1). A large proportion of Methylobacterium ASVs (83 out of 200) were significantly associated with one or either forest (ANOVA) (Fig. 4a; Data Set S1m), regardless of their clade membership. The only exception was clade A1, which was almost exclusively observed (and isolated) (Fig. 3b) in the MSH forest. We found 25 ASVs whose relative abundance significantly increased throughout the growing season (ANOVA; $P<0.05$ ), mostly belonging to clade $A 1$ $(n=11)$. Four ASVs increased significantly in frequency over time in both forests and 
TABLE 2 Summary of statistics from autocorrelation analyses of 179 phyllosphere Methylobacterium samples assessed by rpoB barcoding (200 ASVs) ${ }^{a}$

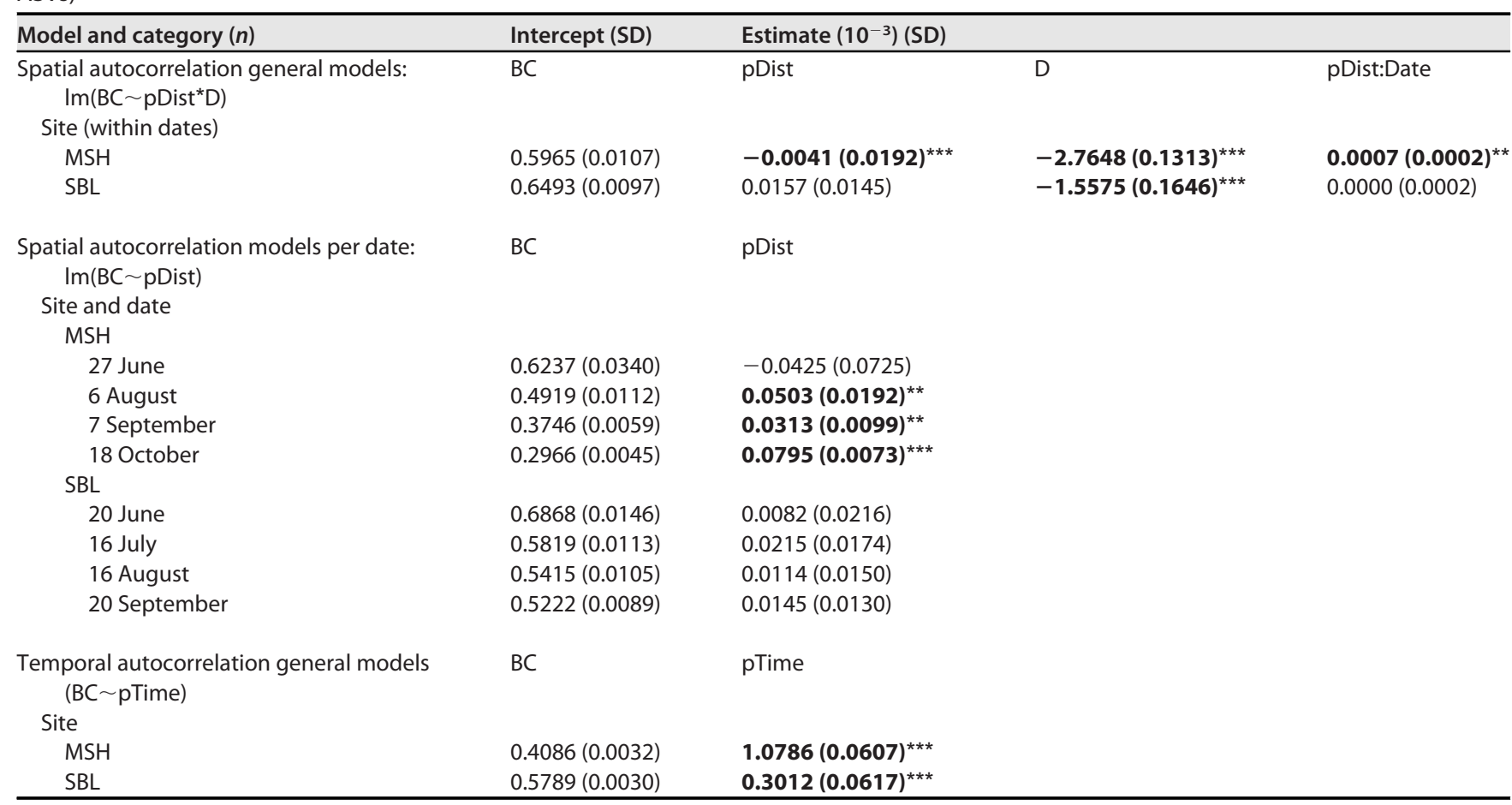

aspatial autocorrelation general models: pairwise dissimilarity between two communities (Bray-Curtis index [BC]) as a function of pairwise spatial distance separating two sampled trees (pDist) and date of sampling (Date [D]) and their interaction (pDist:Date). Spatial autocorrelation models per date: BC as a function of pairwise spatial distance (pDist). Temporal autocorrelation for general models: BC as a function of pairwise spatial time separating two sampled trees ( $p$ Time). For each model, the average and standard deviation of the intercept (mean BC value) are indicated. For each factor (pDist, Date, pDist:Date, and pTime), the average and standard deviation of estimates (slope) are indicated. The significance of estimates was assessed by ANOVA $\left({ }^{* * *}, P<0.001 ;{ }^{* *}, P<0.01 ;{ }^{*}, P<0.05\right)$. Boldface indicates significant estimates.

mostly belonged to group B $(n=3)$ (Data Set S1m). We found no clear association between ASV or clade with host tree species nor plots within forests (data not shown). Methylobacterium diversity was heterogeneously distributed at local spatial scale, as we observed a significant increase of community dissimilarity (Bray-Curtis index [BC]) with geographical distance separating two samples within MSH (spatial autocorrelation analysis; ANOVA; $P<0.001$ ) but not SBL ( $P>0.05$ ) (Table 2; Fig. 4b). We also observed a significant increase in community dissimilarity over time separating two sampling dates in both forests (temporal autocorrelation analysis; $P<0.001$ ) (Table 2), indicating that community composition changed during the growing season. This effect was more marked in MSH than in SBL (Fig. 4c). The overall community BC dissimilarity consistently decreased from June to October in both MSH (from 0.624 to 0.297 ) and SBL (from 0.687 to 0.522 ) (Table 2; Fig. 4d), indicating that the observed change of diversity over time resulted from a progressive homogenization of Methylobacterium community between the beginning and the end of the growing season at the scale of a forest, although without affecting its heterogeneous spatial distributions in MSH (Table 2; Fig. 4e). Methylobacterium communities were strongly phylogenetically clustered (Fig. 4f), with all communities containing ASVs that were much more closely related than expected by chance mean standardized effect size of mean nearest taxon distance [SES(MNTD)] ( \pm standard deviation) $=-4.8 \pm 0.9$; all SES(MNTD) $P$ values were $<0.05$ compared with the null model of random community assembly\}. While all communities were strongly phylogenetically clustered, SES(MNTD) differed among host tree species (ANOVA; $F=6.4$, $P<0.001$ ) and forests (ANOVA; $F=10.9, P<0.001$ ) and decreased during the growing season (ANOVA; $F=95.2, P<0.001$ ).

Effect of short-scale temperature variation in combination with other environmental and genetic factors on Methylobacterium growth performances. We measured growth of 79 Methylobacterium isolates (sampled in 2018 in both forests; 


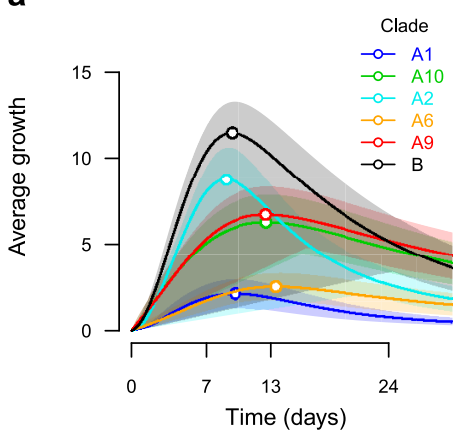

C

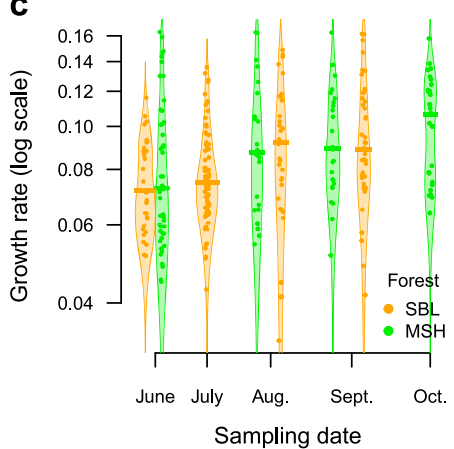

b

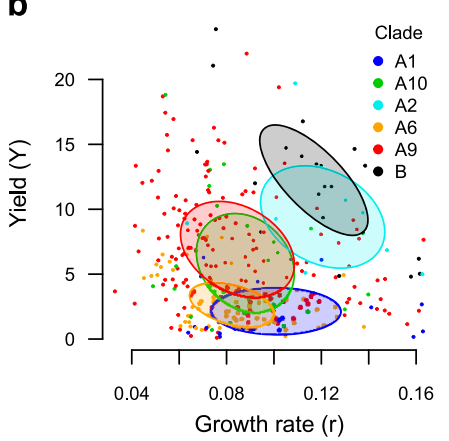

d

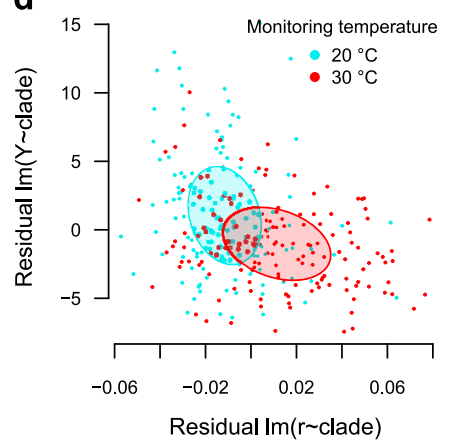

FIG 5 Analysis of growth performance of 79 Methylobacterium isolates under four different temperature treatments. (a) Average growth curves (growth intensity as function of time) for each clade (line, mean value; frame, one-third of standard deviation; point, average maximal growth). (b) Growth rate ( $r$ ) as a function of yield $(Y)$. Each point represents the average $r / Y$ values for an isolate and a temperature treatment (79 isolates times 4 treatments), colored according to clade membership. Ellipsoids are centered on average values per clade and represent $30 \%$ of the confidence interval (standard deviation). (c) $r$ (log scale) as a function of time at which samples from which strains were isolated were collected, colored according to the forest of origin. Points, real data; bars, average $r$ value per forest $(n=2)$ and time $(n=4)$ category. (d) $r$ as a function of $Y$, corrected for clade assignment (residuals of $r$ in function of Clade [ $r \sim$ Clade] and $Y$ in function of Clade [Y Clade] linear regressions). Each point represents the average $r / Y$ residual values for an isolate and a temperature, colored according to the monitoring temperature (legend on top right).

$\mathrm{MSH}, n=32 ; \mathrm{SBL}, n=47$ ) under conditions mimicking temperature variations during the growing season (Fig. S2 to S4; Data Set S1n). Clade membership explained a large part of variation in growth rate $(r)$ and yield $(Y)(7.6 \%$ and $30.6 \%$ of variation explained, respectively; ANOVA; $P<0.001$ ) (Fig. $5 a$ and b and Table 3; Data Set S1o). Group B isolates $(Y=12.2 \pm 5.0)$ have a higher yield than group $\mathrm{A}(Y=5.4 \pm 3.5)$. Isolates from clades $A 1, A 2$, and $B$ had the highest growth rate ( $r$ range, $0.101 \pm 0.032$ to $0.121 \pm 0.031)$. Other clades (A6, A9, and A10) had on average slower growth ( $r$ range, $0.082 \pm 0.021$ to $0.088 \pm 0.024)$. Time of sampling, host tree species, and forest also explained significant variation in growth rate $(5.4 \%, P<0.001 ; 2.2 \%, P<0.01$; and $1.5 \%, P<0.05$, respectively; ANOVA) and limited or no significant variation in yield (1.3\%, $P<0.001 ; 1.3 \%, P<0.01 ; 0.2 \%, P>0.05$, respectively) (Table 3 ). Among the aforementioned factors, only the interaction between time of sampling and clade membership explained significant variation in growth rate $(2.9 \%, P<0.001)$, while all possible pairwise interactions between these factors explained significant variation in yield (range, 1.4 to 5.9\%; $P<0.01$ ) (Table 3). In both SBL and $\mathrm{MSH}$, the growth rate increased consistently from June $(r=0.075 \pm 0.018$ and $0.085 \pm 0.033$, respectively) to September/October $(r=0.097 \pm 0.031$ and $0.103 \pm 0.027$, respectively) (Fig. 5c). The temperature of isolation (at which each isolate was originally isolated) had very limited effect on growth rate $(1.0 \% ; P<0.01)$ and yield $(0.6 \% ; P<0.05)$. These effects were independent of temperatures during preconditioning and monitoring steps (no significant interaction in the ANOVA). The temperature of incubation had significant effects on growth performance. Temperature during the monitoring step explained, respectively, $2.0 \%$ 
TABLE 3 Variance in yield $(Y)$ and growth rate $(r)$ measured in 79 Methylobacterium isolates grown under four temperature treatments ${ }^{a}$

\begin{tabular}{lll}
\hline Factor or interaction & $r$ & $Y$ \\
\hline$F$ & $0.015^{* *}$ & 0.002 \\
$H$ & $0.022^{* * *}$ & $0.013^{* * *}$ \\
$D$ & $0.054^{* * *}$ & $0.013^{* * *}$ \\
$T_{P}$ & 0.001 & $0.014^{* * *}$ \\
$T_{M}$ & $0.158^{* * *}$ & $0.020^{* * *}$ \\
$T_{1}$ & $0.010^{* *}$ & $0.006^{*}$ \\
$C$ & $0.076^{* * *}$ & $0.306^{* * *}$ \\
$F: D$ & 0.005 & $0.035^{* * *}$ \\
$H: D$ & 0.003 & $0.019^{* * *}$ \\
$H: C$ & 0.005 & $0.059^{* * *}$ \\
$D: C$ & $0.029^{* *}$ & $0.028^{* * *}$ \\
$F: C$ & 0.011 & $0.014^{* *}$ \\
$F: T_{1}$ & 0.003 & $0.021^{* * *}$ \\
$H: T_{1}$ & 0.000 & $0.021^{* * *}$ \\
$C: T_{1}$ & $0.024^{* * *}$ & 0.007 \\
F:H:D & $0.012^{* *}$ & $0.023^{* * *}$ \\
F:H:C & $0.010^{* *}$ & 0.002 \\
F:D:C & 0.001 & $0.008^{* *}$ \\
H:D:C & 0.000 & $0.013^{* * *}$ \\
H:D:T & $0.016^{* * *}$ & $0.019^{* * *}$ \\
Other interactions (sum) & 0.177 & 0.079 \\
Residuals & 0.371 & 0.279 \\
\hline Y & &
\end{tabular}

${ }^{a} Y$ and $r$ values were transformed in log to meet normal distribution. Results for the factors clade (C), forest of origin $(F)$, host tree species $(H)$, time of sampling $(D)$, temperature of incubation during preconditioning $\left(T_{p}\right)$ and monitoring $\left(\mathrm{T}_{\mathrm{M}}\right)$ steps, temperature of isolation $\left(\mathrm{T}_{1}\right)$, and their interactions are shown, with significance of $Y$ and $r$ responses indicated ( $\left.{ }^{* * *}, P<0.001 ;{ }^{* *}, P<0.01 ;{ }^{*}, P<0.05\right)$ (see Data set $\mathrm{S} 1 \mathrm{o}$ for details).

and $15.8 \%$ of variation in yield and growth rate $(P<0.01$ and $P<0.001$, respectively; ANOVA) (Table 3), regardless of clade membership, time of sampling, and other environmental factors (no significant interaction in the ANOVA). Isolates incubated at $20^{\circ} \mathrm{C}$ had on average higher yield $(Y=6.9 \pm 5.4)$ but slower growth $(r=0.077 \pm 0.022)$ than isolates incubated at $30^{\circ} \mathrm{C}(Y=4.9 \pm 3.6 ; r=0.100 \pm 0.030)$ (Fig. $\left.5 \mathrm{~d}\right)$. Temperature during the preconditioning step had no effect on growth rate $(P>0.05$; ANOVA) and limited effect on yield (1.4\%; $P<0.05$; ANOVA) (Table 3 ).

\section{DISCUSSION}

Methylobacterium is ubiquitous on leaves in the temperate forests of Québec, and its diversity in this habitat is quite similar to what has been described in the phyllosphere throughout the world, with three main clades, A9 (M. brachiatum, M. pseudosasicola), A6 (related to M. cerastii), and A1 (related to M. gossipicola), dominating diversity. Our barcoding approach based on a clade-specific rpoB marker revealed previously undocumented diversity within these clades, as well as within several other clades that were not detected by a classical $16 \mathrm{~S}$ rRNA gene marker: B (related to $M$. extorquens), A2 (related to M. bullatum and M. marchantiae), A4 (related to M. gnaphalii and M. brachytecii), and A10 (related to $M$. komagatae). This diversity, like that of the overall phyllosphere community, was mostly determined by differences between forests, with barcoding approaches suggesting combined effects of restricted migration, local adaptation to host tree species, and climatic conditions at large geographical scales $(>100$ $\mathrm{km})$. With higher molecular resolution, we observed that Methylobacterium diversity was spatially structured even at the scale of a forest (within $1.2 \mathrm{~km}$ ) and also showed a clear pattern of temporal dynamics and succession over the course of a growing season. This result indicates that although representing a stable proportion of the plant leaf microbiota between years (22), Methylobacterium diversity is highly dynamic within the course of a season. A finer analysis of Methylobacterium diversity suggested that clade identity partly explained Methylobacterium geographical distribution 
at large scales (between forests) but not at finer scales (plots), nor was it an indicator of adaptation to a particular host tree species nor a determinant of temporal dynamics. These results are consistent with previous observations that geographic origin is a stronger driver of phyllosphere Methylobacterium diversity than host identity (22). The distribution of Methylobacterium diversity at small temporal and geographical scales likely resulted from more contemporaneous community assembly events selecting for phenotypic traits that evolved among deeply diverging lineages of Methylobacterium, as has been observed in other bacterial (16) and plant clades (47). We found further evidence for deterministic community assembly, as Methylobacterium communities were strongly phylogenetically clustered compared to the expectation under a stochastic model of community assembly, indicating that the leaf habitat acts as an ecological filter selecting for a nonrandom subset of Methylobacterium diversity.

We explored mechanisms explaining the temporal dynamics of Methylobacterium diversity at the scale of a growing season. Because we observed contrasting Methylobacterium culturable diversity between $20^{\circ} \mathrm{C}$ and $30^{\circ} \mathrm{C}$, we suspected that adaptation to temperature variation during the growing season could explain part of these temporal dynamics. By monitoring Methylobacterium isolate growth under different temperature treatments, we confirmed that temperature affected isolate growth performances but, interestingly, independently from the temperature at which isolates were obtained. The fact that most tested isolates also grew slower but more efficiently at $20^{\circ} \mathrm{C}$ than at $30^{\circ} \mathrm{C}$ (Fig. $5 \mathrm{~d}$ ), regardless of their phylogenetic and environmental characteristics, is in line with a temperaturedependent trade-off between growth rate and yield described for many bacteria (reviewed in reference 48). High-yield strategies are typical of cooperative bacterial populations, while fast-growth strategies are typical of competitive populations (48). These observations also stress the importance of considering incubation temperature when interpreting results from previous culture-based assessments of Methylobacterium diversity.

We provide two lines of evidence that factors other than direct adaptation to temperature drive Methylobacterium responses to temperature variation, by affecting their growth strategy in different competitive conditions rather than by affecting their metabolism directly. First, clade identity was one of the main predictors of overall isolate performance, with some clades (A1, A2, B) possessing a rapid growth strategy under all temperature conditions, while others (clades A6, A9, A10) had systematically slower growth. These cladespecific growth strategies could explain why certain Methylobacterium isolates are less competitive and less frequently isolated at higher temperatures. Still, we cannot rule out that the clade-specific growth strategy also reflects experimental conditions. Second, we observed strong associations between isolate growth performance and time of sampling, regardless of clade membership, suggesting that growth strategies also respond to seasonal variations in environmental conditions and to the level of establishment and competition in the phyllosphere community (48). These associations are unlikely to be driven by the direct effects of temperature on metabolic rates, because isolation temperature had little effect on growth strategies, in contrast to clade identity and time of sampling, which had more significant effects. Together, these observations could explain why isolates from clades $A 1$ and B with fast-growth strategies consistently increase in frequency during this period due to changes in selection for different ecological strategies, leading to the homogenization of the community.

Taken together, our temporal survey of diversity dynamics and screening for growth performance suggests the following timeline of the dynamics of the Methylobacterium phyllosphere community. At the very beginning of the growing season, a pool of bacteria with mixed ecological strategies and genotypes colonizes newly emerging leaves. Due to the stochasticity of this colonization, we initially observe strong dissimilarity among phyllosphere communities, regardless of their spatial position. During the summer, conditions allow the progressive establishment of a diverse Methylobacterium community with a high-yield strategy (48), dominated by increasingly closely phylogenetically related strains. At the end of the growing season, with migration, environmental conditions shifting, and leaves senescing, isolates with a fast-growth strategy are able to grow rapidly, dominating 
the phyllosphere community and leading to its further homogenization before leaves fully senesce. This scenario provides an explanation for the observation of community convergence and increasing homogeneity of phyllosphere communities throughout the growing season $(49,50)$.

Our study illustrates that Methylobacterium is a complex group of divergent lineages with different ecological strategies and distributions, reflecting long-term adaptation to contrasting local environments. Based upon a similar observation, some authors recently proposed the reclassification of Methylobacterium group B within a new genus (Methylorubrum), which they argue is ecologically and evolutionarily distinct from other Methylobacterium clades (31). Although clade B was well supported as a distinct clade in our analyses, our results suggest that it is in fact embedded within clade $A$, which would render the genus Methylobacterium paraphyletic if clade $B$ is defined as a distinct genus (see Fig. S1 in the supplemental material). Furthermore, group B was not particularly ecologically distinct in comparison with other major clades (Fig. 1). Our results emphasize the fact that thorough genomic investigations are needed to clarify the taxonomic status of Methylobacterium. Beyond any taxonomic considerations, neither clade identity assessed by individual genetic markers nor the tremendous ecological diversity among Methylobacterium clades can predict all of the spatial and temporal variation in Methylobacterium diversity in nature. In order to define the niches of Methylobacterium clades and to understand the metabolic mechanisms underlying their contrasting life strategies, future characterization of their functions and genome structure is required using phylogenomic approaches.

In conclusion, we find that Methylobacterium adaptive responses to local environmental variation in the phyllosphere are driven by both long-term inherited ecological strategies that differ among major clades within the genus and by seasonal changes affecting habitat characteristics and community structure in the phyllosphere habitat. Overall, our study, combining culture-free and culture-based approaches, provides novel insights into the factors driving fine-scale adaptation of microbes to their habitats. In the case of Methylobacterium, our approach revealed the particular importance of considering organismal life history strategies to help understand the fine-scale diversity and dynamics of this ecologically important taxon.

\section{MATERIALS AND METHODS}

Phylogenetics of plant-associated Methylobacterium diversity. We evaluated the known Methylobacterium diversity and its distribution across biomes, with a special emphasis on the phyllosphere. First, we constructed a phylogeny of Methylobacteriaceae from the complete nucleotide sequence of $r p o B$, a highly polymorphic housekeeping gene commonly used to reconstruct robust phylogenies in bacteria, because it is unlikely to experience horizontal gene transfer or copy number variation $(51,52)$. We retrieved $r p o B$ sequences from genomes publicly available in September 2020, including 153 Methylobacteria, 30 Microvirga, and 2 Enterovirga members (see Data Set S1a in the supplemental material), performed alignment, and inferred a consensus phylogeny with MrBayes v.3.2.7a (53) (Text S1). For each Methylobacterium reference genome, we retrieved the species name and the sampling origin, when available. Additionally, we assigned each genome to a group (A, B, C) according to previously proposed subdivisions (31). We subdivided group A into nine clades (A1 to A9) (Text S1).

Study sites and sample collection. The two study forests were located at the Gault Nature Reserve (Mont Saint-Hilaire, QC, Canada; 45.54 N 73.16 W), here referred to as MSH, an old forest occupying Mount Saint-Hilaire, and the Station Biologique des Laurentides (Saint-Hippolyte, QC, Canada; $45.99 \mathrm{~N}$ $73.99 \mathrm{~W}$ ), here referred to as SBL, a mosaic of natural wetlands and xeric and mesic forests (Fig. 2; Data Set S1b). In August 2017, for the purpose of a pilot survey, we collected leaves from the subcanopy (3 to $5 \mathrm{~m}$ ) of 19 trees among dominant species in MSH (Fagus grandifolia, Acer saccharum, Acer pensylvanicum, and Ostrya virginiana). In 2018, we realized a time series survey in MSH and SBL. In each forest, we marked and collected leaf samples from the subcanopy of 40 trees (representative of local tree species diversity) in 4 to 6 plots distributed along a 1.2-km transect (Text S1). In MSH, the transect followed an elevation and floristic gradient dominated by tree species F. grandifolia (FAGR), A. saccharum (ACSA), $O$. virginiana (OSVI), and Quercus rubra (QURU). In SBL, the transect followed a constant environment dominated by A. saccharum, F. grandifolia, A. pensylvanicum (ACPE), Abies balsamea (ABBA), and Acer rubrum (ACRU). For this time series, each tree was sampled 3 to 4 times from June to October 2018. For each sampled plot and time point, we also sampled a negative control consisting of empty sterile bags opened and sealed on site. The leaf surface microbial community from each sample was collected with phosphate buffer and split into two equal volumes for microbial community DNA extraction and Methylobacterium isolation, respectively (Text S1). 
Methylobacterium isolation and development of a fine-scale single-copy molecular marker specific to Methylobacterium. For both pilot and time series surveys, we performed Methylobacterium isolation on minimal mineral salts (MMS) synthetic solid medium with $0.1 \%$ methanol supplemented with yeast extract and vitamins (Text S1). For each leaf sample, isolation was replicated at $20^{\circ} \mathrm{C}$ and $30^{\circ} \mathrm{C}$ to minimize biases toward mesophyllic strains. Isolates from the 2017 pilot survey $(n=80)$ (Data Set S1c) were identified by PCR amplification and partial sequencing of the 16S rRNA gene and assigned to Methylobacterium clades (Text S1; Data Set S1d and e). As an alternative to the 16S rRNA gene, we developed a highly polymorphic marker targeting the Methylobacteriaceae family. We tested two candidate genes, rpoB $(51,52,54,55)$ and sucA (55-57), which, in contrast to the 16S rRNA gene, were single copy in Methylobacterium genomes and were polymorphic enough to distinguish among Methylobacterium groups and clades (Text S1; Fig. S1). In 20 representative Methylobacterium isolates from the 2017 pilot survey (Data Set S1c, d, and e), we successfully amplified a rpoB hypervariable region (targeted by primers Met02-352-F and Met02-1121-R), which we chose as a specific marker for Methylobacteriaceae (Text S1; Table S1). Isolates from the 2018 timeline survey $(n=167)$ (Data Set S1e and f) were assigned to Methylobacterium clades using a consensus phylogenetic tree inferred with MrBayes v.3.2.7a (53) from nucleotide sequences of the $r p o B$ marker obtained for these isolates, aligned together with rpoB complete nucleotide sequences available from 188 Methylobacteriaceae genomes (Data Set S1a) and partial nucleotide sequences obtained from 20 representative isolates from the pilot survey (Text S1).

Culture-based assessment of Methylobacterium diversity in the tree phyllosphere. We tested for associations between Methylobacterium culture-based diversity at different phylogenetic depths, with isolate characteristics as proxy for an adaptive response to environmental variables through their evolution, using the $r p o B$ phylogenetic tree built from timeline survey isolates as a guide. We assigned Methylobacterium isolates according to their phylogenetic placement. After exclusion of nodes supported by less than $30 \%$ of bootstraps, the tree was converted into an ultrametric tree scaled proportionally to pairwise nucleotide similarity (PS) (Text S1). First, for each PS value in the tree in the range of 0.926 to 1.000 (corresponding to PS range within clades), we classified isolates into discrete taxa and performed a PERMANOVA (10,000 permutations) on Methylobacterium community dissimilarity using the Bray-Curtis index $(B C)$ based on taxon absolute abundance (Hellinger transformation) using the $R$ package vegan (58). We tested for the relative contribution of four factors and their interactions on taxon frequency: sampling forest (F), temperature of isolation (T), sampling time (D), and host tree species $(\mathrm{H})$. Second, we asked specifically which nodes within the tree were associated with $\mathrm{F}$ and T. For each node with at least $30 \%$ of support and each factor, we tested for the association between embedded taxa and $\mathrm{F}(\mathrm{SBL}$ and $\mathrm{MSH})$ or $\mathrm{T}\left(20\right.$ and $\left.30^{\circ} \mathrm{C}\right)$ by permutation of factors between embedded nodes (100,000 permutations per node) (Text S1).

Culture-free assessment of Methylobacterium diversity in the tree phyllosphere (barcoding). We evaluated the bacterial phyllosphere diversity through barcoding and sequencing of phyllosphere samples from the 2018 timeline survey. First, we evaluated the bacterial diversity targeting the 16S rRNA gene (59) in 46 phyllosphere samples from 13 trees from both forests sampled 3 to 4 times throughout the 2018 growth season. We included one negative control and one positive control consisting of mixed DNAs of Methylobacterium isolates typical of the phyllosphere (METH community) (Text S1). Second, we evaluated the Methylobacteriaceae phyllosphere diversity targeting the rpoB marker (see above) in 184 phyllosphere samples from 53 trees representative of diversity found in MSH $(n=26)$ and SBL $(n=27)$, sampled 3 to 4 times throughout the 2018 growth season. We included four negative controls and four positive controls (METH community). Library preparation and sequencing were performed as described in Text S1. For each phyllosphere sample and controls, we estimated bacterial diversity based on amplicon sequence variants (ASVs) using the package dada2 in R (60). We assessed ASV taxonomy using the SILVA v.138 database for the 16S rRNA gene (61) and a rpoB nucleotide sequence database available for Bacteria (52), curated by a ML phylogenetic tree (200 permutations) (Text S1). Taxonomy for Methylobacterium ASVs (at the clade level) was refined using a BLAST search against NCBI databases for the 16S rRNA gene (62) and using phylogenetic placement for $r p o B$ (Text S1). To validate the $r p o B$ barcoding accuracy in estimating Methylobacterium diversity, we compared Methylobacterium clade relative abundances estimated from $16 \mathrm{~S}$ rRNA gene and $r p o B$ barcoding in a heatmap (Text S1). We also compared Methylobacterium diversity estimations from $r p o B$ barcoding and culture-dependant approaches by matching $r p o B$ partial nucleotide sequences obtained from isolates with those obtained from ASVs (Text S1). We evaluated relative contributions of sampling forest $(F)$, plot within forest $(P)$, host tree species $(H)$, time of sampling $(D)$, and their interactions on bacteria (16S rRNA gene barcoding) and Methylobacteriaceae ( $r p o B$ barcoding) community dissimilarity among phyllosphere samples (BC index, Hellinger transformation on ASV relative abundance), using PERMANOVA (10,000 permutations) (Text S1) and principal-component analysis (PCA). For rpoB barcoding, specifically, we reported Methylobacterium ASV significantly associated with the aforementioned factors $(F, P$, $\mathrm{H}, \mathrm{T}$; ANOVA) into the PCA (Text S1).

Spatial and temporal dynamics of Methylobacterium communities. We evaluated the spatial and temporal dynamics of Methylobacterium communities in the timeline survey ( $r p o B$ barcoding) using autocorrelation analyses. In order to remove potential differences in community composition between forests, we analyzed samples from MSH and SBL separately. For each pairwise comparison between two samples from the same forest, we evaluated the effects of spatial distance (pDist) separating trees sampled at the same date (spatial autocorrelation analyses) and time (pTime) separating dates at which trees were sampled (temporal autocorrelation analyses) on BC dissimilarity among samples (see above). We evaluated the effects of pDist and pTime on BC dissimilarity under linear models by ANOVA (Text S1).

Ecophylogenetic structure of Methylobacterium communities. We quantified the ecophylogenetic structure of Methylobacterium communities by comparing the phylogenetic dissimilarity of cooccurring rpo $B$ 
ASVs with the dissimilarity expected under a null model of stochastic community assembly from the pool of all ASVs, in order to quantify the evidence for different community assembly processes (63) as a function of forest, host tree species, and time of sampling. For each community of Methylobacterium ASVs, we calculated a measure of phylogenetic dissimilarity among cooccurring ASVs (mean nearest taxon distance [MNTD]) and compared the observed MNTD to that expected under a null model of stochastic community assembly from the pool of all ASVs. We calculated the standardized effect size (SES) of MNTD (64), which expresses the difference between the observed MNTD value and the mean and standard deviation of MNTD values obtained across 999 random draws of ASVs from the pool of observed ASVs across all samples while maintaining observed sample ASV richness (65). We evaluated the effects of forest, host tree species, and time of sampling on SES(MNTD) by ANOVA.

Monitoring of Methylobacterium growth performance. We evaluated the growth abilities of 79 Methylobacterium isolates from the timeline survey for four temperature treatments mimicking temperature variations during the growing season. Each treatment consisted of an initial preconditioning step ( $P$ step) during which each isolate was incubated on solid MMS medium with methanol as the sole carbon source for 20 days at either $20^{\circ} \mathrm{C}$ (P20) or $30^{\circ} \mathrm{C}$ (P30), and a second monitoring step (M step) during which preconditioned isolates were incubated on the same medium and their growth was monitored for 24 days at $20^{\circ} \mathrm{C}(\mathrm{P} 20 \mathrm{M} 20$ and $\mathrm{P} 20 \mathrm{M} 30)$ or $30^{\circ} \mathrm{C}(\mathrm{P} 30 \mathrm{M} 20$ and P30M30) (Fig. S2). Treatments P20M20 and P30M30 mimicked stable thermal environments, and treatments P20M30 and P30M20 mimicked variable thermal environments. For each isolate and each combination of treatments (PXXMXX), we realized 5 replicates, randomly spotted on 48 petri dishes according to a 6-by- 6 grid. During the monitoring step, we took photographs of each petri dish at days 7, 13, and 24 after inoculation (Fig. S2). Photos were converted to pixel intensities with ImageJ $1.52 \mathrm{e}$ and processed in $\mathrm{R}$ for background correction, measurement of spot intensities, and correction for position-dependent competition effects (Fig. S3; Text S1). For each isolate and temperature treatment, logistic growth curves were inferred from bacterial spot intensity variation observed over three time points during the monitoring step. From growth curves, we estimated maximum growth intensity or yield $(Y)$ and growth rate $(r)$ as the inverse of lag plus log time necessary to reach $Y(48,66)$ (Fig. S4; Text S1). We evaluated the effects of the following factors on Methylobacterium growth abilities ( $Y$ and $r$ ) under different temperature treatments: isolate assignment to clades $(C)$, forest of origin (F), host tree species $(H)$, time of sampling (D), temperature of isolation $\left(T_{i}\right.$; at which each isolate was isolated), temperature of incubation during preconditioning $\left(T_{p}\right)$ and monitoring $\left(T_{M}\right)$ steps, and all possible interactions between those factors (ANOVA) (Text S1).

Data availability. Raw reads for $16 \mathrm{~S}$ rRNA gene and $r p o B$ barcoding on phyllosphere communities (BioProject PRJNA729807; BioSamples SAMN19164946 to SAMN19165146) were deposited in NCBI under SRA accession numbers SRR14532212 to SRR14532451. Partial nucleotide sequences from marker genes obtained by SANGER sequencing on Methylobacterium isolates (BioProject PRJNA730554; Biosamples SAMN19190155 to SAMN19190401) were deposited in NCBI under GenBank accession numbers MZ268514 to MZ268593 (16S rRNA gene), MZ330152 to MZ330358 (rpoB gene), and MZ330130 to MZ330151 (sucA). BioProject, BioSample, SRA, and GenBank accession numbers are listed in Data Set S1. R code and related data were deposited on Github (https://github.com/JBLED/methylo-phyllo-diversity).

\section{SUPPLEMENTAL MATERIAL}

Supplemental material is available online only.

DATA SET S1, XLSX file, 1.5 MB.

TEXT S1, PDF file, $0.3 \mathrm{MB}$.

FIG S1, PDF file, $0.2 \mathrm{MB}$.

FIG S2, PDF file, $0.1 \mathrm{MB}$.

FIG S3, PDF file, $0.2 \mathrm{MB}$.

FIG S4, PDF file, $0.4 \mathrm{MB}$.

FIG S5, PDF file, $0.5 \mathrm{MB}$.

TABLE S1, DOCX file, $0.1 \mathrm{MB}$.

TABLE S2, DOCX file, $0.1 \mathrm{MB}$.

\section{ACKNOWLEDGMENTS}

This research received funding from FRQNT, NSERC, Canada Research Chairs, and the NSF (grant no. DEB-1831838).

We thank Geneviève Lajoie, Dominique Tardif, Ariane Lafrenière, Hélène DionPhénix, Yves Terrat, and Kenta Araya for help with sampling, Sylvain Dallaire for help with the phenotyping screen, Sergey Stolyar for discussions, and Gault Natural Reserve (McGill University), Station Biologique des Laurentides (UdeM), Centre d'Étude de la Forêt (CEF), QCBS, and two anonymous reviewers for their helpful suggestions.

J.-B.L., E.S.-L., D.C.-M., G.B., B.J.S., and S.W.K. planned field work and the experiments. J.-B.L. E.S.-L., D.C.-M., and G.B. performed the experiments. J.-B.L., E.S.-L., S.W.K., D.S., and J.M.S performed the bioinformatic analyses. J.A.F., C.J.M., and J.M.S. provided discussion 
in the early stages of this study. J.-B.L., B.J.S., and S.W.K. drafted the manuscript with

contributions from C.J.M.

\section{REFERENCES}

1. Vorholt JA. 2012. Microbial life in the phyllosphere. Nat Rev Microbiol 10: 828-840. https://doi.org/10.1038/nrmicro2910.

2. Lindow SE, Brandl MT. 2003. Microbiology of the phyllosphere. Appl Environ Microbiol 69:1875-1883. https://doi.org/10.1128/AEM.69.4.1875-1883 .2003 .

3. Fürnkranz M, Wanek W, Richter A, Abell G, Rasche F, Sessitsch A. 2008. Nitrogen fixation by phyllosphere bacteria associated with higher plants and their colonizing epiphytes of a tropical lowland rainforest of Costa Rica. ISME J 2:561-570. https://doi.org/10.1038/ismej.2008.14.

4. Laforest-Lapointe I, Messier C, Kembel SW. 2016. Host species identity, site and time drive temperate tree phyllosphere bacterial community structure. Microbiome 4:27. https://doi.org/10.1186/s40168-016-0174-1.

5. Laforest-Lapointe I, Messier C, Kembel SW. 2016. Tree phyllosphere bacterial communities: exploring the magnitude of intra- and inter-individual variation among host species. PeerJ 4:e2367. https://doi.org/10.7717/peerj.2367.

6. Noble AS, Noe S, Clearwater MJ, Lee CK. 2020. A core phyllosphere microbiome exists across distant populations of a tree species indigenous to New Zealand. PLoS One 15:e0237079. https://doi.org/10.1371/ journal.pone.0237079.

7. Kembel SW, O'Connor TK, Arnold HK, Hubbell SP, Wright SJ, Green JL. 2014. Relationships between phyllosphere bacterial communities and plant functional traits in a neotropical forest. Proc Natl Acad Sci U S A 111 : 13715-13720. https://doi.org/10.1073/pnas.1216057111.

8. Shade A, Gregory Caporaso J, Handelsman J, Knight R, Fierer N. 2013. A meta-analysis of changes in bacterial and archaeal communities with time. ISME J 7:1493-1506. https://doi.org/10.1038/ismej.2013.54.

9. Malik AA, Martiny JBH, Brodie EL, Martiny AC, Treseder KK, Allison SD. 2020. Defining trait-based microbial strategies with consequences for soil carbon cycling under climate change. ISME J 14:1-9. https://doi.org/10 .1038/s41396-019-0510-0.

10. Moyes AB, Kueppers LM, Pett-Ridge J, Carper DL, Vandehey N, O'Neil J, Frank AC. 2016. Evidence for foliar endophytic nitrogen fixation in a widely distributed subalpine conifer. New Phytol 210:657-668. https://doi .org/10.1111/nph.13850

11. Lajoie G, Kembel SW. 2019. Making the most of trait-based approaches for microbial ecology. Trends Microbiol 27:814-823. https://doi.org/10 .1016/j.tim.2019.06.003.

12. Nemergut DR, Schmidt SK, Fukami T, O'Neill SP, Bilinski TM, Stanish LF, Knelman JE, Darcy JL, Lynch RC, Wickey P, Ferrenberg S. 2013. Patterns and processes of microbial community assembly. Microbiol Mol Biol Rev 77:342-356. https://doi.org/10.1128/MMBR.00051-12.

13. Cavender-Bares J, Kozak KH, Fine PVA, Kembel SW. 2009. The merging of community ecology and phylogenetic biology. Ecol Lett 12:693-715. https://doi.org/10.1111/j.1461-0248.2009.01314.x.

14. Martiny JBH, Jones SE, Lennon JT, Martiny AC. 2015. Microbiomes in light of traits: a phylogenetic perspective. Science 350:aac9323. https://doi .org/10.1126/science.aac9323.

15. Tromas N, Taranu ZE, Castelli M, Pimentel JSM, Pereira DA, Marcoz R, Shapiro BJ, Giani A. 2020. The evolution of realized niches within freshwater Synechococcus. Environ Microbiol 22:1238-1250. https://doi.org/ 10.1111/1462-2920.14930.

16. Tromas N, Taranu ZE, Martin BD, Willis A, Fortin N, Greer CW, Shapiro BJ. 2018. Niche separation increases with genetic distance among bloomforming cyanobacteria. Front Microbiol 9:438. https://doi.org/10.3389/ fmicb.2018.00438.

17. Thompson LR, Sanders JG, McDonald D, Amir A, Ladau J, Locey KJ, Prill RJ, Tripathi A, Gibbons SM, Ackermann G, Navas-Molina JA, Janssen S, Kopylova E, Vázquez-Baeza Y, González A, Morton JT, Mirarab S, Zech Xu Z, Jiang L, Haroon MF, Kanbar J, Zhu Q, Jin Song S, Kosciolek T, Bokulich NA, Lefler J, Brislawn CJ, Humphrey G, Owens SM, Hampton-Marcell J, Berg-Lyons D, McKenzie V, Fierer N, Fuhrman JA, Clauset A, Stevens RL, Shade A, Pollard KS, Goodwin KD, Jansson JK, Gilbert JA, Knight R, Earth Microbiome Project Consortium. 2017. A communal catalogue reveals Earth's multiscale microbial diversity. Nature 551:457-463. https://doi .org/10.1038/nature24621.

18. Poretsky R, Rodriguez-R LM, Luo C, Tsementzi D, Konstantinidis KT. 2014 Strengths and limitations of $16 \mathrm{~S}$ rRNA gene amplicon sequencing in revealing temporal microbial community dynamics. PLoS One 9:e93827. https://doi.org/10.1371/journal.pone.0093827.

19. Ranjan R, Rani A, Metwally A, McGee HS, Perkins DL. 2016. Analysis of the microbiome: advantages of whole genome shotgun versus $16 \mathrm{~S}$ amplicon sequencing. Biochem Biophys Res Commun 469:967-977. https://doi.org/10 .1016/j.bbrc.2015.12.083.

20. Corpe WA, Rheem S. 1989. Ecology of the methylotrophic bacteria on living leaf surfaces. FEMS Microbiol Ecol 62:243-249. https://doi.org/10.1111/j.1574 -6968.1989.tb03698.x.

21. Keppler F, Hamilton JTG, Brass M, Röckmann T. 2006. Methane emissions from terrestrial plants under aerobic conditions. Nature 439:187-191. https://doi.org/10.1038/nature04420.

22. Knief C, Ramette A, Frances L, Alonso-Blanco C, Vorholt JA. 2010. Site and plant species are important determinants of the Methylobacterium community composition in the plant phyllosphere. ISME J 4:719-728. https:// doi.org/10.1038/ismej.2010.9.

23. Clarke PH. 1983. The biochemistry of methylotrophs by C. Anthony Academic Press; London, New York, 1982 xvi +432 pages. £24.00, \$49.50. FEBS Lett 160:303-303. https://doi.org/10.1016/0014-5793(83)80989-2.

24. Anthony C. 1991. Assimilation of carbon by methylotrophs. Biotechnology 18:79-109. https://doi.org/10.1016/b978-0-7506-9188-8.50011-5.

25. Ivanova EG, Doronina NV, Trotsenko YA. 2001. Aerobic methylobacteria are capable of synthesizing auxins. Microbiology 70:392-397. https://doi .org/10.1023/A:1010469708107.

26. Madhaiyan M, Poonguzhali S, Lee HS, Hari K, Sundaram SP, Sa TM. 2005. Pink-pigmented facultative methylotrophic bacteria accelerate germination, growth and yield of sugarcane clone Co86032 (Saccharum officinarum L.). Biol Fertil Soils 41:350-358. https://doi.org/10.1007/s00374-005 -0838-7.

27. Madhaiyan M, Poonguzhali S, Sa T. 2007. Metal tolerating methylotrophic bacteria reduces nickel and cadmium toxicity and promotes plant growth of tomato (Lycopersicon esculentum L.). Chemosphere 69:220-228. https://doi .org/10.1016/j.chemosphere.2007.04.017.

28. Dourado MN, Aparecida Camargo Neves A, Santos DS, Araújo WL. 2015 Biotechnological and agronomic potential of endophytic pink-pigmented methylotrophic Methylobacterium spp. Biomed Res Int 2015:909016. https://doi.org/10.1155/2015/909016.

29. Ryu JH, Chungbuk NU, Madhaiyan M, Chungbuk NU, Poonguzhali S, Chungbuk NU, Yim WJ, Chungbuk NU, Indiragandhi $P$, Chungbuk NU, Kim KA, Chungbuk NU, Anandham R, Chungbuk NU, Yun JC, Kim KH, Sa TM. 2006. Plant growth substances produced by Methylobacterium spp. and their effect on tomato (Lycopersicon esculentum L.) and red pepper (Capsicum annuum L.) growth. J Microbiol Biotechnol 16:1622-1628.

30. Lee HS, Madhaiyan M, Kim CW, Choi SJ, Chung KY, Sa TM. 2006. Physiological enhancement of early growth of rice seedlings (Oryza sativa L.) by production of phytohormone of $\mathrm{N}_{2}$-fixing methylotrophic isolates. Biol Fertil Soils 42:402-408. https://doi.org/10.1007/s00374-006-0083-8.

31. Green PN, Ardley JK. 2018. Review of the genus Methylobacterium and closely related organisms: a proposal that some Methylobacterium species be reclassified into a new genus, Methylorubrum gen. nov. Int J Syst Evol Microbiol 68:2727-2748. https://doi.org/10.1099/ijsem.0.002856.

32. Chen W-M, Cai C-Y, Li Z-H, Young C-C, Sheu S-Y. 2019. Methylobacterium oryzihabitans sp. nov., isolated from water sampled from a rice paddy field. Int J Syst Evol Microbiol 69:3843-3850. https://doi.org/10.1099/ ijsem.0.003693.

33. Feng G-D, Chen W, Zhang X-J, Zhang J, Wang S-N, Zhu H. 2020. Methylobacterium nonmethylotrophicum sp. nov., isolated from tungsten mine tailing. Int J Syst Evol Microbiol 70:2867-2872. https://doi.org/10.1099/ ijsem.0.004112.

34. Juan Jia L, Zhang K, Shuai Tang K, Yu Meng J, Zheng C, Ying Feng F. 2020. Methylobacterium crusticola sp. nov., isolated from biological soil crusts. Int J Syst Evol Microbiol 70:2089-2095.

35. Kim J, Chhetri G, Kim I, Lee B, Jang W, Kim MK, Seo T. 2020. Methylobacterium terricola $\mathrm{sp}$. nov., a gamma radiation-resistant bacterium isolated from gamma ray-irradiated soil. Int J Syst Evol Microbiol 70:2449-2456. https://doi.org/10.1099/ijsem.0.004054. 
36. Kim J, Chhetri G, Kim I, Kim MK, Seo T. 2020. Methylobacterium durans sp. nov., a radiation-resistant bacterium isolated from gamma ray-irradiated soil. Antonie Van Leeuwenhoek 113:211-220. https://doi.org/10.1007/ s10482-019-01331-2.

37. Ten LN, Li W, Elderiny NS, Kim MK, Lee S-Y, Rooney AP, Jung H-Y. 2020. Methylobacterium segetis sp. nov., a novel member of the family Methylobacteriaceae isolated from soil on Jeju Island. Arch Microbiol 202:747-754. https:// doi.org/10.1007/s00203-019-01784-z.

38. Jiang L, An D, Wang X, Zhang K, Li G, Lang L, Wang L, Jiang C, Jiang Y. 2020. Methylobacterium planium sp. nov., isolated from a lichen sample. Arch Microbiol 202:1709-1715. https://doi.org/10.1007/s00203-020-01881-4.

39. Pascual JA, Ros M, Martínez J, Carmona F, Bernabé A, Torres R, Lucena T, Aznar R, Arahal DR, Fernández F. 2020. Methylobacterium symbioticum sp. nov., a new species isolated from spores of Glomus iranicum var. tenuihypharum. Curr Microbiol 77:2031-2041. https://doi.org/10.1007/s00284-020 $-02101-4$.

40. Marx CJ, Bringel F, Chistoserdova L, Moulin L, Farhan UI Haque $M$, Fleischman DE, Gruffaz C, Jourand P, Knief C, Lee M-C, Muller EEL, Nadalig T, Peyraud R, Roselli S, Russ L, Goodwin LA, Ivanova N, Kyrpides N, Lajus A, Land ML, Médigue C, Mikhailova N, Nolan M, Woyke T, Stolyar S, Vorholt JA, Vuilleumier S. 2012. Complete genome sequences of six strains of the genus Methylobacterium. J Bacteriol 194:4746-4748. https://doi.org/10 .1128/JB.01009-12.

41. Tani A, Ogura Y, Hayashi T, Kimbara K. 2015. Complete genome sequence of Methylobacterium aquaticum strain $22 \mathrm{~A}$, isolated from Racomitrium japonicum moss. Genome Announc 3:e00266-15. https://doi.org/10.1128/genomeA .00266-15.

42. Minami T, Ohtsubo $Y$, Anda $M$, Nagata $Y$, Tsuda $M$, Mitsui $H$, Sugawara $M$, Minamisawa K. 2016. Complete genome sequence of Methylobacterium sp. strain AMS5, an isolate from a soybean stem. Genome Announc 4:e00144-16. https://doi.org/10.1128/genomeA.00144-16.

43. Morohoshi T, Ikeda T. 2016. Complete genome sequence of Methylobacterium populi $\mathrm{P}-1 \mathrm{M}$, isolated from pink-pigmented household biofilm. Genome Announc 4:e00458-16. https://doi.org/10.1128/genomeA.00458-16.

44. Belkhelfa S, Labadie K, Cruaud C, Aury J-M, Roche D, Bouzon M, Salanoubat M, Döring V. 2018. Complete genome sequence of the facultative methylotroph Methylobacterium extorquens TK 0001 isolated from soil in Poland. Genome Announc 6:e00018-18. https://doi.org/10.1128/ genomeA.00018-18.

45. Green PN. 2015. Methylobacterium, p 1-8. In Bergey's manual of systematics of Archaea and Bacteria. Wiley, Hoboken, NJ.

46. Charron G, Leducq JB, Bertin C, Dube AK, Landry CR. 2014. Exploring the northern limit of the distribution of Saccharomyces cerevisiae and Saccharomyces paradoxus in North America. FEMS Yeast Res 14:281-288. https://doi .org/10.1111/1567-1364.12100.

47. Cavender-Bares J, Ackerly DD, Baum DA, Bazzaz FA. 2004. Phylogenetic overdispersion in Floridian oak communities. Am Nat 163:823-843. https://doi .org/10.1086/386375.

48. Lipson DA. 2015. The complex relationship between microbial growth rate and yield and its implications for ecosystem processes. Front Microbiol 6:615. https://doi.org/10.3389/fmicb.2015.00615.

49. Copeland JK, Yuan L, Layeghifard M, Wang PW, Guttman DS. 2015. Seasonal community succession of the phyllosphere microbiome. Mol Plant Microbe Interact 28:274-285. https://doi.org/10.1094/MPMI-10-14-0331-FI.

50. Maignien L, DeForce EA, Chafee ME, Eren AM, Simmons SL. 2014. Ecological succession and stochastic variation in the assembly of Arabidopsis thaliana phyllosphere communities. mBio 5:e00682-13. https://doi.org/10 $.1128 / \mathrm{mBio} .00682-13$.

51. Vos M, Quince C, Pijl AS, de Hollander M, Kowalchuk GA. 2012. A Comparison of rpoB and $16 \mathrm{~S}$ rRNA as markers in pyrosequencing studies of bacterial diversity. PLoS One 7:e30600. https://doi.org/10.1371/journal .pone.0030600.

52. Ogier J-C, Pagès S, Galan M, Barret M, Gaudriault S. 2019. rpoB, a promising marker for analyzing the diversity of bacterial communities by amplicon sequencing. BMC Microbiol 19:171. https://doi.org/10.1186/s12866 -019-1546-z.

53. Ronquist F, Teslenko M, van der Mark P, Ayres DL, Darling A, Höhna S, Larget B, Liu L, Suchard MA, Huelsenbeck JP. 2012. MrBayes 3.2: efficient Bayesian phylogenetic inference and model choice across a large model space. Syst Biol 61:539-542. https://doi.org/10.1093/sysbio/sys029.

54. Küpfer M, Kuhnert P, Korczak BM, Peduzzi R, Demarta A. 2006. Genetic relationships of Aeromonas strains inferred from 16S rRNA, gyrB and rpoB gene sequences. Int J Syst Evol Microbiol 56:2743-2751. https://doi.org/ 10.1099/ijs.0.63650-0.

55. Ee R, Madhaiyan M, Ji L, Lim Y-L, Nor NM, Tee K-K, Chen J-W, Yin W-F. 2016. Chania multitudinisentens gen. nov., sp. nov., an $\mathrm{N}$-acyl-homoserine-lactone-producing bacterium in the family Enterobacteriaceae isolated from landfill site soil. Int J Syst Evol Microbiol 66:2297-2304. https:// doi.org/10.1099/ijsem.0.001025.

56. Frank RAW, Price AJ, Northrop FD, Perham RN, Luisi BF. 2007. Crystal structure of the E1 component of the Escherichia coli 2-oxoglutarate dehydrogenase multienzyme complex. J Mol Biol 368:639-651. https://doi .org/10.1016/j.jmb.2007.01.080.

57. Bell RL, González-Escalona N, Stones R, Brown EW. 2011. Phylogenetic evaluation of the 'Typhimurium' complex of Salmonella strains using a seven-gene multi-locus sequence analysis. Infect Genet Evol 11:83-91. https://doi.org/10.1016/j.meegid.2010.10.005.

58. Dixon P. 2003. VEGAN, a package of $R$ functions for community ecology. J Vegetation Science 14:927-930. https://doi.org/10.1111/j.1654-1103.2003 .tb02228.x.

59. Redford AJ, Bowers RM, Knight R, Linhart Y, Fierer N. 2010. The ecology of the phyllosphere: geographic and phylogenetic variability in the distribution of bacteria on tree leaves. Environ Microbiol 12:2885-2893. https:// doi.org/10.1111/j.1462-2920.2010.02258.x.

60. Callahan BJ, McMurdie PJ, Rosen MJ, Han AW, Johnson AJA, Holmes SP. 2016. DADA2: high resolution sample inference from Illumina amplicon data. Nat Methods 13:581-583. https://doi.org/10.1038/nmeth.3869.

61. Quast C, Pruesse E, Yilmaz P, Gerken J, Schweer T, Yarza P, Peplies J, Glöckner FO. 2013. The SILVA ribosomal RNA gene database project: improved data processing and web-based tools. Nucleic Acids Res 41: D590-D596. https://doi.org/10.1093/nar/gks1219.

62. Boratyn GM, Camacho C, Cooper PS, Coulouris G, Fong A, Ma N, Madden TL, Matten WT, McGinnis SD, Merezhuk Y, Raytselis Y, Sayers EW, Tao T, Ye J, Zaretskaya I. 2013. BLAST: a more efficient report with usability improvements. Nucleic Acids Res 41:W29-W33. https://doi.org/10.1093/nar/gkt282.

63. Stegen JC, Lin X, Fredrickson JK, Chen X, Kennedy DW, Murray CJ, Rockhold ML, Konopka A. 2013. Quantifying community assembly processes and identifying features that impose them. ISME J 7:2069-2079. https://doi.org/10.1038/ismej.2013.93.

64. Kembel SW, Cowan PD, Helmus MR, Cornwell WK, Morlon H, Ackerly DD, Blomberg SP, Webb CO. 2010. Picante: R tools for integrating phylogenies and ecology. Bioinformatics 26:1463-1464. https://doi.org/10 $.1093 /$ bioinformatics/btq166.

65. Kembel SW. 2009. Disentangling niche and neutral influences on community assembly: assessing the performance of community phylogenetic structure tests. Ecol Lett 12:949-960. https://doi.org/10.1111/j.1461-0248.2009.01354.x.

66. Zwietering MH, Jongenburger I, Rombouts FM, van 't Riet K. 1990. Modeling of the bacterial growth curve. Appl Environ Microbiol 56:1875-1881. https://doi.org/10.1128/aem.56.6.1875-1881.1990. 